

Т. Х. Расулов, Исследование существенного спектра одного матричного оператора, ТМФ, 2010, том 164, номер 1, 62-77

DOI: https://doi.org/10.4213/tmf6524

Использование Общероссийского математического портала Math-Net.Ru подразумевает, что вы прочитали и согласны с пользовательским соглашением http://www.mathnet.ru/rus/agreement

Параметры загрузки:

IP : 54.224 .60 .19

26 апреля 2023 г., 16:07:49






\title{
ИССЛЕДОВАНИЕ СУЩЕСТВЕННОГО СПЕКТРА ОДНОГО МАТРИЧНОГО ОПЕРАТОРА
}

\begin{abstract}
Рассмотрен матричный оператор $H$, соответствующий системе с несохраняющимся ограниченным числом частиц на решетке. Описана структура существенного спектра оператора $H$. Доказано, что существенный спектр этого оператора состоит из объединения не более чем четырех отрезков.
\end{abstract}

Ключевые слова: матричный оператор, система с несохраняющимся ограниченным числом частиц, пространство Фока, обобщенная модель Фридрихса, существенный спектр, собственное значение.

\section{1. ВВЕДЕНИЕ}

Настоящая статья является продолжением работы [1], в которой рассмотрен матричный оператор $H$, соответствующий системе с несохраняющимся ограниченным числом частиц на решетке, доказано, что его существенный спектр совпадает со спектром оператора канала, и выделены “двухчастичная" и “трехчастичная" ветви существенного спектра. Кроме того, в указанной работе получен аналог уравнения Фаддеева для собственных функций оператора $H$. В настоящей работе мы докажем, что существенный спектр этого оператора состоит из объединения не более чем четырех отрезков и изучим расположение этих отрезков.

Отметим, что данный матричный оператор $H$ представляет собой некомпактное возмущение оператора $H_{0}$, рассмотренного в работах [2]-[5], и является симметричным вариантом матричного оператора из статьи [6]. Положение существенного спектра оператора $H$ и аналог уравнения Фаддеева для собственных функций в более общем случае изучены в работе [7]. Оператор $H$ также можно представить как решетчатый аналог операторов, рассмотренных в работах [8], [9].

Существенный спектр операторов, соответствующих системам с сохраняющимся ограниченным числом частиц, т.е. операторов Шредингера, заданных на непрерывном пространстве и на решетке, изучался во многих работах (см., например, труды [10]-[13]). В частности, в работе [12] показано, что существенный спектр

*Бухарский государственный университет, Бухара, Узбекистан. E-mail: rth@mail.ru 
четырехчастичного оператора Шредингера на решетке состоит из объединения не более чем четырех отрезков, а в работе [13] доказано, что существенный спектр оператора Шредингера на решетке состоит из объединения не более чем конечного числа отрезков даже в случае, когда соответствующий двухчастичный оператор Шредингера на решетке имеет бесконечное число собственных значений.

Структура настоящей статьи следующая. В разделе 2 мы вводим матричный оператор $H$ как ограниченный самосопряженный оператор в гильбертовом пространстве, формулируем задачу и представляем основные результаты работы. В разделе 3 мы рассматриваем некоторые спектральные свойства обобщенной модели Фридрихса; при выполнении одного естественного условия исследование этих свойств может быть сведено к анализу спектральных свойств операторов с более простой структурой. В разделе 4 мы выводим аналог уравнения Фаддеева для собственных функций оператора $H$. Раздел 5 посвящен доказательству основных результатов.

Следует отметить, что в непрерывном случае “двухчастичная" и “трехчастичная" ветви непрерывного спектра операторов, рассмотренных в работах [8], [9], представляют собой полубесконечные прямые и пересекаются. В нашем случае "двухчастичная" и "трехчастичная" ветви существенного спектра матричного оператора $H$ заполняют отрезки конечной длины и могут не иметь точек пересечения, другими словами, в спектре возникает лакуна. Теорема 3 утверждает, что такие лакуны существуют при некоторых естественных условиях.

\section{2. ПОСТАНОВКА ЗАДАЧИ И ФОРМУЛИРОВКА ОСНОВНЫХ РЕЗУЛЬТАТОВ}

2.1. Матричный оператор. Пусть $\mathbb{T}^{3} \equiv(-\pi ; \pi]^{3}-$ трехмерный куб с отождествлением противоположных граней, $\left(\mathbb{T}^{3}\right)^{2}=\mathbb{T}^{3} \times \mathbb{T}^{3}-$ декартово произведение, $L_{2}\left(\mathbb{T}^{3}\right)$ - гильбертово пространство квадратично-интегрируемых (комплекснозначных) функций, определенных на $\mathbb{T}^{3}, L_{2}^{\mathrm{s}}\left(\left(\mathbb{T}^{3}\right)^{2}\right)$ - гильбертово пространство квадратично-интегрируемых симметричных (комплекснозначных) функций, определенных на $\left(\mathbb{T}^{3}\right)^{2}, \mathbb{C}$ - комплексная плоскость. Пусть $\mathcal{H}=\mathcal{H}_{0} \oplus \mathcal{H}_{1} \oplus \mathcal{H}_{2}$ - прямая сумма пространств $\mathcal{H}_{0}=\mathbb{C}, \mathcal{H}_{1}=L_{2}\left(\mathbb{T}^{3}\right)$ и $\mathcal{H}_{2}=L_{2}^{\mathrm{s}}\left(\left(\mathbb{T}^{3}\right)^{2}\right)$. Гильбертово пространство $\mathcal{H}$ называется трехчастичным обрезанным подпространством пространства Фока.

Рассмотрим матричный оператор $H$, действующий в гильбертовом пространстве $\mathcal{H}$ и задающийся как

$$
H=\left(\begin{array}{ccc}
H_{00} & H_{01} & 0 \\
H_{10} & H_{11} & H_{12} \\
0 & H_{21} & H_{22}
\end{array}\right)
$$


где операторы $H_{i j}: \mathcal{H}_{j} \rightarrow \mathcal{H}_{i}, i, j=0,1,2$, определяются по формулам

$$
\begin{aligned}
\left(H_{00} f_{0}\right)_{0} & =w_{0} f_{0}, \quad\left(H_{01} f_{1}\right)_{0}=\int v_{0}(s) f_{1}(s) d s, \quad\left(H_{10} f_{0}\right)_{1}(p)=v_{0}(p) f_{0}, \\
\left(H_{11} f_{1}\right)_{1}(p) & =w_{1}(p) f_{1}(p), \quad\left(H_{12} f_{2}\right)_{1}(p)=\int v_{1}(s) f_{2}(p, s) d s \\
\left(H_{21} f_{1}\right)_{2}(p, q) & =\frac{v_{1}(p) f_{1}(q)+v_{1}(q) f_{1}(p)}{2}, \\
\left(H_{22} f_{2}\right)_{2}(p, q) & =w_{2}(p, q) f_{2}(p, q)-v_{2}(p) \int v_{2}(s) f_{2}(s, q) d s- \\
& -v_{2}(q) \int v_{2}(s) f_{2}(p, s) d s
\end{aligned}
$$

Здесь $f_{i} \in \mathcal{H}_{i}, w_{0}$ - фиксированное вещественное число, $w_{1}(\cdot), v_{i}(\cdot)$ - вещественнозначные непрерывные (ненулевые) функции на $\mathbb{T}^{3}, i=0,1,2$, а $w_{2}(\cdot, \cdot)$ - вещественнозначная непрерывная симметричная функция на $\left(\mathbb{T}^{3}\right)^{2}$. Здесь и в дальнейшем интеграл без указания пределов означает интегрирование по всей области изменения переменных интегрирования. В современной математической физике операторы $H_{01}$ и $H_{12}$ называются операторами уничтожения, а операторы $H_{10}$ и $H_{21}-$ операторами рождения. Отметим, что в рассматриваемой системе число рождений и уничтожений частиц равно единице, т.е. $H_{i j}=0$ при $|i-j|>1$.

Легко можно проверить, что оператор $H$, определенный матрицей (1) и действующий в гильбертовом пространстве $\mathcal{H}$, ограничен и самосопряжен.

2.2. Основные понятия. Введем ограниченный и самосопряженный оператор (из семейства операторов обобщенной моделей Фридрихса) $h(p), p \in \mathbb{T}^{3}$, действующий в $\mathcal{H}_{0} \oplus \mathcal{H}_{1}$ по формуле

$$
h(p)=\left(\begin{array}{cc}
h_{00}(p) & h_{01} \\
h_{10} & h_{11}(p)
\end{array}\right)
$$

где

$$
\begin{aligned}
\left(h_{00}(p) f_{0}\right)_{0} & =w_{1}(p) f_{0}, & \left(h_{01} f_{1}\right)_{0} & =\frac{1}{\sqrt{2}} \int v_{1}(s) f_{1}(s) d s, \\
\left(h_{10} f_{0}\right)_{1}(q) & =\frac{v_{1}(q) f_{0}}{\sqrt{2}}, & h_{11}(p) & =h_{11}^{0}(p)-v, \\
\left(h_{11}^{0}(p) f_{1}\right)_{1}(q) & =w_{2}(p, q) f_{1}(q), & \left(v f_{1}\right)_{1}(q) & =v_{2}(q) \int v_{2}(s) f_{1}(s) d s .
\end{aligned}
$$

Пусть

$$
m=\min _{p, q \in \mathbb{T}^{3}} w_{2}(p, q), \quad M=\max _{p, q \in \mathbb{T}^{3}} w_{2}(p, q)
$$

и $\sigma_{\text {disc }}(h(p))$ - дискретный спектр оператора $h(p), p \in \mathbb{T}^{3}$. 
Положим

$$
\Sigma=\bigcup_{p \in \mathbb{T}^{3}} \sigma_{\mathrm{disc}}(h(p)) \cup[m ; M] .
$$

Следующая теорема описывает положение существенного спектра оператора $H$.

Tеорема 1. Для существенного спектра $\sigma_{\text {ess }}(H)$ оператора $H$ имеет место равенство $\sigma_{\text {ess }}(H)=\Sigma$.

ОПРЕДЕЛЕНИЕ. Множества

$$
\sigma_{\text {two }}(H)=\bigcup_{p \in \mathbb{T}^{3}} \sigma_{\text {disc }}(h(p)), \quad \sigma_{\text {three }}(H)=[m ; M]
$$

называются "двухчастичной" и "трехчастичной" ветвями существенного спектра оператора $H$.

Сформулируем следующее важное для дальнейших рассуждений условие на функции в (2).

УСловиЕ. Пусть функция $v(\cdot)=v_{1}(\cdot) v_{2}(\cdot)$ является нечетной на $\mathbb{T}^{3}$, а функция $w_{2}(\cdot, \cdot)$ имеет единственный невырожденный минимум в точке $(0,0) \in\left(\mathbb{T}^{3}\right)^{2}$ и является четной по переменным $p$ и $q$ (в отдельности) на $\mathbb{T}^{3}$.

Отметим, что функция $w_{2}(p, q)=\sum_{i=1}^{3}\left(2-\cos p_{i}-\cos q_{i}\right), p=\left(p_{1}, p_{2}, p_{3}\right) \in \mathbb{T}^{3}$, $q=\left(q_{1}, q_{2}, q_{3}\right) \in \mathbb{T}^{3}$, удовлетворяет этому условию.

При выполнении данного условия дискретный спектр оператора $h(p)$ совпадает с объединением дискретных спектров операторов

$$
h_{1}(p) \equiv h_{11}(p), \quad h_{2}(p)=\left(\begin{array}{cc}
h_{00}(p) & h_{01} \\
h_{10} & h_{11}^{0}(p)
\end{array}\right)
$$

при всех $p \in \mathbb{T}^{3}$ (см. лемму 1 ниже). Тогда справедливо равенство

$$
\sigma_{\text {two }}(H)=\bigcup_{i=1,2} \bigcup_{p \in \mathbb{T}^{3}} \sigma_{\text {disc }}\left(h_{i}(p)\right) \text {. }
$$

Из определения операторов $h_{i}(p), i=1,2$, видно, что они имеют более простую структуру, чем $h(p)$.

Положим

$$
m(p)=\min _{q \in \mathbb{T}^{3}} w_{2}(p, q), \quad M(p)=\max _{q \in \mathbb{T}^{3}} w_{2}(p, q) .
$$

При каждом фиксированном $p \in \mathbb{T}^{3}$ определим регулярные в $\mathbb{C} \backslash[m(p) ; M(p)]$ функции

$$
\Delta_{1}(p ; z)=1-\int \frac{v_{2}^{2}(s) d s}{w_{2}(p, s)-z}, \quad \Delta_{2}(p ; z)=w_{1}(p)-z-\frac{1}{2} \int \frac{v_{1}^{2}(s) d s}{w_{2}(p, s)-z},
$$

являющиеся определителями Фредгольма, ассоциированными с операторами $h_{1}(p)$ и $h_{2}(p)$ соответственно.

3 Теоретическая и математическая физика, т. 164, № 1, 2010 г. 
Для любых $p, q \in \mathbb{T}^{3}$ и $z<m$ справедливо неравенство $w_{2}(p, q)-z>0$, отсюда следует, что

$$
\int \frac{v_{i}^{2}(s) d s}{w_{2}(p, s)-z}>0, \quad i=1,2 .
$$

Так как функция $w_{2}(\cdot, \cdot)$ имеет единственный невырожденный минимум в точке $(0,0) \in\left(\mathbb{T}^{3}\right)^{2}$, а функции $v_{i}(\cdot), i=1,2$, непрерывны на $\mathbb{T}^{3}$, оба интеграла в $(7)$ конечны.

Из теоремы о предельном переходе под знаком интеграла Лебега следует, что $\Delta_{i}(0 ; m)=\lim _{p \rightarrow 0} \Delta_{i}(p ; m)$ и, следовательно, функции $\Delta_{i}(\cdot ; m)$ непрерывны на $\mathbb{T}^{3}$, $i=1,2$. Аналогично функция $\Delta_{2}(\cdot ; M)$ также непрерывна на $\mathbb{T}^{3}$.

\section{3. Основные результаты.}

Теорема 2. Существенный спектр оператора $H$ представляет собой оббединение не более чем четырех отрезков.

Введем следующие обозначения:

$$
\begin{array}{ll}
a_{1}=\min \bigcup_{p \in \mathbb{T}^{3}} \sigma_{\text {disc }}\left(h_{1}(p)\right), & b_{1}=\max \bigcup_{p \in \mathbb{T}^{3}} \sigma_{\text {disc }}\left(h_{1}(p)\right), \\
a_{2}=\min \left\{\bigcup_{p \in \mathbb{T}^{3}} \sigma_{\text {disc }}\left(h_{2}(p)\right) \cap(-\infty ; m]\right\}, & b_{2}=\max \left\{\bigcup_{p \in \mathbb{T}^{3}} \sigma_{\text {disc }}\left(h_{2}(p)\right) \cap(-\infty ; m]\right\}, \\
a_{3}=\min \left\{\bigcup_{p \in \mathbb{T}^{3}} \sigma_{\text {disc }}\left(h_{2}(p)\right) \cap[M ;+\infty)\right\}, & b_{3}=\max \left\{\bigcup_{p \in \mathbb{T}^{3}} \sigma_{\text {disc }}\left(h_{2}(p)\right) \cap[M ;+\infty)\right\} .
\end{array}
$$

Следующая теорема описывает структуру существенного спектра оператора $H$.

Теорема 3. Предположим, что $\min _{p \in \mathbb{T}^{3}} \Delta_{1}(p ; m) \geqslant 0$ и выполняется приведенное в $n .2 .2$ условие. Тогда имеют место следующие утверждения.

1. Пусть $\max _{p \in \mathbb{T}^{3}} \Delta_{2}(p ; M) \leqslant 0$. Тогда:

1.1) если $\min _{p \in \mathbb{T}^{3}} \Delta_{2}(p ; m) \geqslant 0$, mo $\sigma_{\mathrm{ess}}(H)=[m ; M]$;

1.2) если $\min _{p \in \mathbb{T}^{3}} \Delta_{2}(p ; m)<0 u \max _{p \in \mathbb{T}^{3}} \Delta_{2}(p ; m) \geqslant 0$, mo $\sigma_{\mathrm{ess}}(H)=\left[a_{2} ; M\right]$, где $a_{2}<m$

1.3) если $\max _{p \in \mathbb{T}^{3}} \Delta_{2}(p ; m)<0$, mo $\sigma_{\mathrm{ess}}(H)=\left[a_{2} ; b_{2}\right] \cup[m ; M]$, где $b_{2}<m$.

2. Пусть $\min _{p \in \mathbb{T}^{3}} \Delta_{2}(p ; M) \leqslant 0 u \max _{p \in \mathbb{T}^{3}} \Delta_{2}(p ; M)>0$. Тогда:

2.1) если $\min _{p \in \mathbb{T}^{3}} \Delta_{2}(p ; m) \geqslant 0$, mo $\sigma_{\mathrm{ess}}(H)=\left[m ; b_{3}\right]$, где $b_{3}>M$;

2.2) если $\min _{p \in \mathbb{T}^{3}} \Delta_{2}(p ; m)<0 u \max _{p \in \mathbb{T}^{3}} \Delta_{2}(p ; m) \geqslant 0$, mo $\sigma_{\mathrm{ess}}(H)=\left[a_{2} ; b_{3}\right]$, где $a_{2}<m u b_{3}>M$

2.3) если $\max _{p \in \mathbb{T}^{3}} \Delta_{2}(p ; m)<0$, mo $\sigma_{\mathrm{ess}}(H)=\left[a_{2} ; b_{2}\right] \cup\left[m ; b_{3}\right]$, где $b_{2}<m u b_{3}>M$.

3. Пусть $\min _{p \in \mathbb{T}^{3}} \Delta_{2}(p ; M)>0$. Тогда:

3.1) если $\min _{p \in \mathbb{T}^{3}} \Delta_{2}(p ; m) \geqslant 0$, mо $\sigma_{\mathrm{ess}}(H)=[m ; M] \cup\left[a_{3} ; b_{3}\right]$, где $a_{3}>M$;

3.2) если $\min _{p \in \mathbb{T}^{3}} \Delta_{2}(p ; m)<0 u \max _{p \in \mathbb{T}^{3}} \Delta_{2}(p ; m) \geqslant 0$, mo $\sigma_{\mathrm{ess}}(H)=\left[a_{2} ; M\right] \cup$ $\left[a_{3} ; b_{3}\right]$, где $a_{2}<m u a_{3}>M$

3.3) если $\max _{p \in \mathbb{T}^{3}} \Delta_{2}(p ; m)<0$, mo $\sigma_{\mathrm{ess}}(H)=\left[a_{2} ; b_{2}\right] \cup[m ; M] \cup\left[a_{3} ; b_{3}\right]$, где $b_{2}<m$ $u a_{3}>M$. 
ЗАМЕЧАнИЕ 1. Если $\min _{p \in \mathbb{T}^{3}} \Delta_{1}(p ; m)<0$ и $\max _{p \in \mathbb{T}^{3}} \Delta_{1}(p ; m) \geqslant 0$ (или $\left.\max _{p \in \mathbb{T}^{3}} \Delta_{1}(p ; m)<0\right)$ и выполняется условие из п. 2.2 , то можно сформулировать аналогичную теорему, заменив $[m ; M]$ на $\left[a_{1} ; M\right]$, где $a_{1}<m$ (или, соответственно, заменив $[m ; M]$ на $\left[a_{1} ; b_{1}\right] \cup[m ; M]$, где $\left.b_{1}<m\right)$.

ЗАмечание 2. Теорема 3 играет важную роль при нахождении необходимых и достаточных условий конечности (см. работу [4]) или бесконечности (см. работу [5]) числа собственных значений, лежащих вне существенного спектра оператора $H$.

\section{3. НЕКОТОРЫЕ СПЕКТРАЛЬНЫЕ СВОЙСТВА ОПЕРАТОРОВ $H_{I}(P)$}

Пусть оператор $h_{0}(p)$ действует в $\mathcal{H}_{0} \oplus \mathcal{H}_{1}$ как

$$
h_{0}(p)=\left(\begin{array}{cc}
0 & 0 \\
0 & h_{11}^{0}(p)
\end{array}\right), \quad p \in \mathbb{T}^{3} .
$$

Оператор возмущения $h(p)-h_{0}(p)$ оператора $h_{0}(p)$ является ограниченным самосопряженным оператором, ранг которого не выше 3. Следовательно, по известной теореме Вейля (см. книгу [11], теорема XIII.14) о сохранении существенного спектра при возмущениях конечного ранга существенный спектр оператора $h(p)$ совпадает с существенным спектром оператора $h_{0}(p), p \in \mathbb{T}^{3}$. Известно, что $\sigma_{\text {ess }}\left(h_{0}(p)\right)=$ $[m(p) ; M(p)]$, поэтому

$$
\sigma_{\text {ess }}(h(p))=[m(p) ; M(p)] .
$$

Теперь установим связь между собственными значениями операторов $h(p)$ и $h_{i}(p)$, $i=1,2$. Верна следующая

Лемма 1. Пусть выполнено условие из п. 2.2. Тогда для любого $p \in \mathbb{T}^{3}$ число $z \in \mathbb{C} \backslash \sigma_{\mathrm{ess}}(h(p))$ является собственным значением оператора $h(p)$, если и только если z является собственным значением хотя би одного из операторов $h_{i}(p)$, $i=1,2$.

ДОКАЗАТЕЛЬСтво. Пусть число $z \in \mathbb{C} \backslash \sigma_{\text {ess }}(h(p))$ есть собственное значение оператора $h(p)$ и пусть $f=\left(f_{0}, f_{1}\right) \in \mathcal{H}_{0} \oplus \mathcal{H}_{1}$ - соответствующая собственная вектор-функция. Тогда $f_{0}$ и $f_{1}$ удовлетворяют следующей системе уравнений

$$
\begin{aligned}
\left(w_{1}(p)-z\right) f_{0}+\frac{1}{\sqrt{2}} \int v_{1}\left(q^{\prime}\right) f_{1}\left(q^{\prime}\right) d q^{\prime} & =0, \\
\frac{1}{\sqrt{2}} v_{1}(q) f_{0}+\left(w_{2}(p, q)-z\right) f_{1}(q)-v_{2}(q) \int v_{2}\left(q^{\prime}\right) f_{1}\left(q^{\prime}\right) d q^{\prime} & =0 .
\end{aligned}
$$

В силу равенства (8) для любых $z \in \mathbb{C} \backslash \sigma_{\text {ess }}(h(p))$ и $q \in \mathbb{T}^{3}$ справедливо соотношение $w_{2}(p, q)-z \neq 0$ при всех $p \in \mathbb{T}^{3}$. Из второго уравнения этой системы имеем

$$
f_{1}(q)=\frac{v_{2}(q) C_{f_{1}}}{w_{2}(p, q)-z}-\frac{1}{\sqrt{2}} \frac{v_{1}(q) f_{0}}{w_{2}(p, q)-z},
$$


где

$$
C_{f_{1}}=\int v_{2}\left(q^{\prime}\right) f_{1}\left(q^{\prime}\right) d q^{\prime}
$$

Подставляя выражение для $f_{1}$ в первое уравнение системы и равенство (11), учитывая условие из п. 2.2, получим, что система уравнений (9) имеет ненулевое решение тогда и только тогда, когда система уравнений

$$
\Delta_{2}(p ; z) f_{0}=0, \quad \Delta_{1}(p ; z) C_{f_{1}}=0
$$

имеет ненулевое решение, т.е. при условии $\Delta_{1}(p ; z) \Delta_{2}(p ; z)=0$. Если в (4) положить $v_{2}(q) \equiv 0$, то $h(p)=h_{2}(p)$ (ср. формулы $(3)$ и $\left.(5)\right)$, при этом $z \in \mathbb{C} \backslash \sigma_{\mathrm{ess}}(h(p))$ является собственным значением оператора $h_{2}(p)$ тогда и только тогда, когда $\Delta_{2}(p ; z)=0$.

Аналогично можно показать [14], что число $z \in \mathbb{C} \backslash \sigma_{\mathrm{ess}}(h(p))$ является собственным значением оператора $h_{1}(p), p \in \mathbb{T}^{3}$, тогда и только тогда, когда $\Delta_{1}(p ; z)=0$. Лемма доказана.

Для $p \in \mathbb{T}^{3}$ положим $V(p)=h(p)-h_{0}(p)$.

ЛЕмма 2. Для любого фиксированного $p \in \mathbb{T}^{3}$ оператор $V(p)$ имеет не более одного положительного и не более двух отрицательных простых собственных значений.

Доказательство. Уравнение $V(p) f=z f, z \neq 0, f \in \mathcal{H}_{0} \oplus \mathcal{H}_{1}$, на собственные значения оператора $V$ эквивалентно системе уравнений

$$
\left(w_{1}(p)-z\right) f_{0}+\frac{\left(v_{1}, f_{1}\right)_{1}}{\sqrt{2}}=0, \quad \frac{v_{1}(q) f_{0}}{\sqrt{2}}-v_{2}(q)\left(v_{2}, f_{1}\right)_{1}=z f_{1},
$$

где $(\cdot, \cdot)_{1}-$ скалярное произведение в $\mathcal{H}_{1}$. Так как $z \neq 0$, из второго уравнения системы получаем, что

$$
f_{1}(q)=\frac{1}{\sqrt{2}} \frac{v_{1}(q)}{z} f_{0}-\frac{v_{2}(q)}{z} C_{f_{1}},
$$

где $C_{f_{1}}$ определена в формуле $(11)$. Подставляя выражение для $f_{1}$ в первое уравнение системы и в равенство (11), имеем

$$
\begin{aligned}
\left(z^{2}-w_{1}(p) z-\frac{\left\|v_{1}\right\|_{1}^{2}}{2}\right) f_{0}+\frac{\left(v_{1}, v_{2}\right)_{1}}{\sqrt{2}} C_{f_{1}} & =0 \\
\frac{\left(v_{1}, v_{2}\right)_{1}}{\sqrt{2}} f_{0}+\left(z+\left\|v_{2}\right\|_{1}^{2}\right) C_{f_{1}} & =0
\end{aligned}
$$

Здесь через $\|\cdot\|_{1}$ обозначена норма в $\mathcal{H}_{1}$.

Положим для $z \neq 0, p \in \mathbb{T}^{3}$

$$
\begin{aligned}
P_{\left(v_{1}, v_{2}\right)_{1}}(p ; z) & =\left(z^{2}-w_{1}(p) z-\frac{\left\|v_{1}\right\|_{1}^{2}}{2}\right)\left(z+\left\|v_{2}\right\|_{1}^{2}\right)-\frac{\left(v_{1}, v_{2}\right)_{1}^{2}}{2}= \\
& =P_{0}(p ; z)-\frac{\left(v_{1}, v_{2}\right)_{1}^{2}}{2}
\end{aligned}
$$


Система уравнений (14) имеет решение тогда и только тогда, когда детерминант этой системы равен нулю, т.е. когда $P_{\left(v_{1}, v_{2}\right)_{1}}(p ; z)=0$. Таким образом, проблему поиска собственных значений оператора $V(p)$ мы свели к задаче анализа нулей полинома $P_{\left(v_{1}, v_{2}\right)_{1}}(p ; \cdot)$ степени 3 .

Пользуясь неравенством $\left|\left(v_{1}, v_{2}\right)_{1}\right| \leqslant\left\|v_{1}\right\|_{1}\left\|v_{2}\right\|_{1}$, получим

$$
P_{0}(p ; z) \geqslant P_{\left(v_{1}, v_{2}\right)_{1}}(p ; z) \geqslant P_{\left\|v_{1}\right\|_{1}\left\|v_{2}\right\|_{1}}(p ; z),
$$

где

$$
P_{\left\|v_{1}\right\|_{1}\left\|v_{2}\right\|_{1}}(p ; z)=P_{0}(p ; z)-\frac{\left\|v_{1}\right\|_{1}^{2}\left\|v_{2}\right\|_{1}^{2}}{2} .
$$

Возможны три случая: $v_{1}(\cdot)$ и $v_{2}(\cdot)$ ортогональны, $v_{1}(\cdot)$ и $v_{2}(\cdot)$ параллельны, $v_{1}(\cdot)$ и $v_{2}(\cdot)$ не ортогональны и не параллельны. Рассмотрим каждый из этих случаев в отдельности.

Пусть $v_{1}(\cdot)$ и $v_{2}(\cdot)$ ортогональны. Тогда

$$
P_{0}(p ; z)=P_{\left(v_{1}, v_{2}\right)_{1}}(p ; z)>P_{\left\|v_{1}\right\|_{1}\left\|v_{2}\right\|_{1}}(p ; z) .
$$

В этом случае числа

$$
\begin{aligned}
& \widehat{z}_{1}(p)=-\left\|v_{2}\right\|_{1}^{2}<0, \quad \widehat{z}_{2}(p)=\frac{w_{1}(p)-\sqrt{w_{1}^{2}(p)+2\left\|v_{1}\right\|_{1}^{2}}}{2}<0, \\
& \widehat{z}_{3}(p)=\frac{w_{1}(p)+\sqrt{w_{1}^{2}(p)+2\left\|v_{1}\right\|_{1}^{2}}}{2}>0
\end{aligned}
$$

являются нулями полинома $P_{\left(v_{1}, v_{2}\right)_{1}}(p ; \cdot)=P_{0}(p ; \cdot)$, т.е. собственными значениями оператора $V(p)$ для каждого $p \in \mathbb{T}^{3}$. Отметим, что числа $\widehat{z}_{n}(p), n=1,2,3$, являются нулями полинома $P_{0}(p ; \cdot)$ и в случае, когда $v_{1}(\cdot)$ и $v_{2}(\cdot)$ не ортогональны.

Пусть $v_{1}(\cdot)$ и $v_{2}(\cdot)$ параллельны. Тогда

$$
P_{0}(p ; z)>P_{\left(v_{1}, v_{2}\right)_{1}}(p ; z)=P_{\left\|v_{1}\right\|_{1}\left\|v_{2}\right\|_{1}}(p ; z) .
$$

В этом случае полином $P_{\left(v_{1}, v_{2}\right)_{1}}(p ; \cdot)$ записывается в виде

$$
P_{\left(v_{1}, v_{2}\right)_{1}}(p ; z)=z\left(z^{2}-z\left(\left\|v_{2}\right\|_{1}^{2}-w_{1}(p)\right)-\left(\frac{\left\|v_{1}\right\|_{1}^{2}}{2}+w_{1}(p)\left\|v_{2}\right\|_{1}^{2}\right)\right) .
$$

Отсюда видно, что числа

$$
\begin{aligned}
& \widetilde{z}_{1}(p)=0, \quad \widetilde{z}_{2}(p)=\frac{\left\|v_{2}\right\|_{1}^{2}-w_{1}(p)-\sqrt{\left(\left\|v_{2}\right\|_{1}^{2}+w_{1}(p)\right)^{2}+2\left\|v_{1}\right\|_{1}^{2}}}{2}<0, \\
& \widetilde{z}_{3}(p)=\frac{\left\|v_{2}\right\|_{1}^{2}-w_{1}(p)+\sqrt{\left(\left\|v_{2}\right\|_{1}^{2}+w_{1}(p)\right)^{2}+2\left\|v_{1}\right\|_{1}^{2}}}{2}>0
\end{aligned}
$$


являются нулями полинома $P_{\left(v_{1}, v_{2}\right)_{1}}(p ; \cdot)=P_{\left\|v_{1}\right\|_{1}\left\|v_{2}\right\|_{1}}(p ; \cdot)$, т.е. собственными значениями оператора $V(p)$ для каждого $p \in \mathbb{T}^{3}$. Заметим, что числа $\widetilde{z}_{n}(p), n=1,2,3$, являются нулями полинома $P_{\left\|v_{1}\right\|_{1}\left\|v_{2}\right\|_{1}}(p ; \cdot)$ и в случае, когда $v_{1}(\cdot)$ и $v_{2}(\cdot)$ не параллельны.

Пусть $v_{1}(\cdot)$ и $v_{2}(\cdot)$ не ортогональны и не параллельны. Тогда

$$
P_{0}(p ; z)>P_{\left(v_{1}, v_{2}\right)_{1}}(p ; z)>P_{\left\|v_{1}\right\|_{1}\left\|v_{2}\right\|_{1}}(p ; z) .
$$

Положим $a_{1}(p)=\min \left\{\widehat{z}_{1}(p), \widehat{z}_{2}(p)\right\}, a_{2}(p)=\max \left\{\widehat{z}_{1}(p), \widehat{z}_{2}(p)\right\}$. Без ограничения общности мы можем считать, что для любого $p \in \mathbb{T}^{3}$ справедливы неравенства $\widetilde{z}_{2}(p)<\widehat{z}_{2}(p), \widetilde{z}_{2}(p)<\widehat{z}_{1}(p), \widehat{z}_{3}(p)<\widetilde{z}_{3}(p)$ (в противном случае применяется аналогичный метод). Тогда отсюда следует, что

$$
\widetilde{z}_{2}(p)<a_{1}(p) \leqslant a_{2}(p)<\widetilde{z}_{1}(p)=0<\widehat{z}_{3}(p)<\widetilde{z}_{3}(p) .
$$

Так как числа $\widetilde{z}_{2}(p)$ и $a_{1}(p)$ являются нулями полиномов $P_{\left\|v_{1}\right\|_{1}\left\|v_{2}\right\|_{1}}(p ; \cdot)$ и $P_{0}(p ; \cdot)$ соответственно, имеем

$$
\begin{gathered}
P_{\left(v_{1}, v_{2}\right)_{1}}\left(p ; \widetilde{z}_{2}(p)\right)>P_{\left\|v_{1}\right\|_{1}\left\|v_{2}\right\|_{1}}\left(p ; \widetilde{z}_{2}(p)\right)=0, \\
0=P_{0}\left(p ; a_{1}(p)\right)<P_{\left(v_{1}, v_{2}\right)_{1}}\left(p ; a_{1}(p)\right),
\end{gathered}
$$

т.е. на границе сегмента $\left[\widetilde{z}_{2}(p), a_{1}(p)\right]$ полином $P_{\left(v_{1}, v_{2}\right)_{1}}(p ; \cdot)$ имеет разные знаки. Следовательно, существует точка $z_{1}(p) \in\left(\widetilde{z}_{2}(p), a_{1}(p)\right)$ такая, что $P_{\left(v_{1}, v_{2}\right)_{1}}\left(p ; z_{1}(p)\right)=0$. Аналогично можно показать, что существуют числа $z_{2}(p) \in\left(a_{2}(p), \widetilde{z}_{1}(p)\right)$ и $z_{3}(p) \in$ $\left(\widehat{z}_{3}(p), \widetilde{z}_{3}(p)\right)$, которые являются нулями полинома $P_{\left(v_{1}, v_{2}\right)_{1}}(p ; \cdot)$. Так как $P_{\left(v_{1}, v_{2}\right)_{1}}(p ; \cdot)$ есть полином третьей степени, эти нули являются простыми. Видно, что $z_{1}(p)<$ $z_{2}(p)<0$ и $z_{3}(p)>0$. Лемма доказана.

Лемма 3. Для любого $p \in \mathbb{T}^{3}$ имеет место равенство $\sigma_{\mathrm{ess}}(V(p))=\{0\}$.

ДоказАтельство. Для любого $p \in \mathbb{T}^{3}$ рассмотрим уравнение $V(p) f=0$ относительно $f \in \mathcal{H}_{0} \oplus \mathcal{H}_{1}$, которое эквивалентно системе уравнений

$$
w_{1}(p) f_{0}+\frac{\left(v_{1}, f_{1}\right)_{1}}{\sqrt{2}}=0, \quad \frac{v_{1}(q) f_{0}}{\sqrt{2}}-v_{2}(q)\left(v_{2}, f_{1}\right)_{1}=0 .
$$

Можно показать, что элементы подпространства

$$
L_{0}=\left\{f=\left(0, f_{1}\right) \in \mathcal{H}_{0} \oplus \mathcal{H}_{1}:\left(v_{i}, f_{1}\right)_{1}=0, \quad i=1,2\right\}
$$

являются решениями системы уравнений (15). Видно, что $\operatorname{dim} L_{0}=+\infty$. Это означает, что $0 \in \sigma_{\mathrm{ess}}(V(p))$.

Теперь докажем обратное включение, т.е. что $\sigma_{\mathrm{ess}}(V(p)) \subset\{0\}$ для любого $p \in \mathbb{T}^{3}$. Пусть $z$ - произвольная точка множества $\sigma(V(p)) \backslash\{0\}$. Тогда $z \in \sigma_{\text {disc }}(V(p))$ (см. доказательство леммы 2). Следовательно,

$$
\sigma(V(p)) \backslash\{0\} \subset \sigma_{\text {disc }}(V(p))=\sigma(V(p)) \backslash \sigma_{\text {ess }}(V(p)) .
$$

Это означает, что включение $\sigma_{\mathrm{ess}}(V(p)) \subset\{0\}$ выполняется для любого $p \in \mathbb{T}^{3}$. Лемма доказана. 
Из доказанной леммы вытекает, что для любого $p \in \mathbb{T}^{3}$ оператор $V(p)$ может иметь только ненулевые конечнократные собственные значения, а число $z=0$ является бесконечнократным собственным значением оператора $V(p)$.

Введем обозначения

$$
|V(p)|=\left(V^{2}(p)\right)^{1 / 2}, \quad V_{ \pm}(p)=\frac{V(p) \pm|V(p)|}{2}
$$

где $\left(V^{2}(p)\right)^{1 / 2}$ - неотрицательный квадратный корень из оператора $V^{2}(p)$. Тогда $V_{+}(p) \geqslant 0, V_{-}(p) \leqslant 0$ и $V(p)=V_{+}(p)+V_{-}(p)$. Так как операторы $V_{+}(p)$ и $-V_{-}(p)$ неотрицательны, существуют неотрицательные квадратные корни $V_{+}^{1 / 2}(p)$ и $\left(-V_{-}\right)^{1 / 2}(p)$ из этих операторов. Для любого фиксированного $z<m(p)(z>M(p))$ оператор $h_{0}(p)-z I+V_{+}(p)$ (оператор $\left.h_{0}(p)-z I+V_{-}(p)\right)$ является обратимым и положительным (отрицательным); здесь $I$ - единичный оператор в $\mathcal{H}_{0} \oplus \mathcal{H}_{1}$. Пусть

$$
\begin{array}{ll}
r_{+}(p ; z)=\left(h_{0}(p)-z I+V_{+}(p)\right)^{-1}, & z<m(p), \\
r_{-}(p ; z)=\left(-\left(h_{0}(p)-z I+V_{-}(p)\right)\right)^{-1}, & z>M(p),
\end{array}
$$

и $r_{ \pm}^{1 / 2}(p ; z)$ - положительные квадратные корни из $r_{ \pm}(p ; z)$. Обозначим через $N_{-}(p ; z)$ (через $\left.N_{+}(p ; z)\right)$ число собственных значений оператора $h_{2}(p)$, лежащих левее $z<m(p)$ (правее $z>M(p))$.

Пусть дан ограниченный самосопряженный оператор $A$, действующий в гильбертовом пространстве $\mathcal{H}$, не имеющий точек существенного спектра правее $z$. Обозначим через $\mathcal{H}_{A}(z)$ такое подпространство, что $(A f, f)>z(f, f)$ для любого $f \in \mathcal{H}_{A}(z)$ и положим $n(z, A)=\sup _{\mathcal{H}_{A}(z)} \operatorname{dim} \mathcal{H}_{A}(z)$. По определению чисел $N_{-}(p ; z)$ и $N_{+}(p ; z)$ имеем

$$
\begin{aligned}
N_{-}(p ; z) & =n(-z,-h(p)), & -z>-m(p), \\
N_{+}(p ; z) & =n(z, h(p)), & z>M(p) .
\end{aligned}
$$

Следующая лемма является реализацией известного принципа Бирмана-Швингера для оператора $h(p)$ (см. работы [5], [14]).

Лемма 4. Для любого $z<m(p)$ оператор $\left(-V_{-}\right)^{1 / 2}(p) r_{+}(p ; z)\left(-V_{-}\right)^{1 / 2}(p)$ является компактным и

$$
N_{-}(p ; z)=n\left(1,\left(-V_{-}\right)^{1 / 2}(p) r_{+}(p ; z)\left(-V_{-}\right)^{1 / 2}(p)\right)
$$

ДокАзАтельство. Оператор $\left(-V_{-}\right)^{1 / 2}(p)$ имеет конечный ранг, а оператор $r_{+}(p ; z)\left(-V_{-}\right)^{1 / 2}(p)$ является ограниченным, отсюда следует, что оператор $\left(-V_{-}\right)^{1 / 2}(p) r_{+}(p ; z)\left(-V_{-}\right)^{1 / 2}(p)$ компактен.

Запишем оператор $h(p)$ как $h(p)=h_{0}(p)+V_{+}(p)+V_{-}(p)$. Пусть элемент $u \in$ $\mathcal{H}_{-h(p)}(-z)$, т.е. $\left(\left(h_{0}(p)-z I+V_{+}(p)\right) u, u\right)<\left(\left(-V_{-}(p)\right) u, u\right)$. Тогда

$$
\left(r_{+}^{1 / 2}(p ; z)\left(-V_{-}(p)\right) r_{+}^{1 / 2}(p ; z) g, g\right)>0, \quad g=\left(h_{0}(p)-z I+V_{-}(p)\right)^{1 / 2} u
$$


Следовательно, $N_{-}(p ; z) \leqslant n\left(1, r_{+}^{1 / 2}(p ; z)\left(-V_{-}(p)\right) r_{+}^{1 / 2}(p ; z)\right)$. Аналогично можно получить обратное неравенство. Учитывая эти факты, имеем, что

$$
N_{-}(p ; z)=n\left(1, r_{+}^{1 / 2}(p ; z)\left(-V_{-}(p)\right) r_{+}^{1 / 2}(p ; z)\right) .
$$

Если операторы $T_{1}$ и $T_{2}$ ограничены, то все нетривиальные собственные значения и их кратность для операторов $T_{1} T_{2}$ и $T_{2} T_{1}$ совпадают (см., например, монографию $[15])$, дискретный спектр оператора $r_{+}^{1 / 2}(p ; z)\left(-V_{-}(p)\right) r_{+}^{1 / 2}(p ; z)$ совпадает с дискретным спектром оператора $\left(-V_{-}\right)^{1 / 2}(p) r_{+}(p ; z)\left(-V_{-}\right)^{1 / 2}(p)$. Следовательно,

$$
n\left(1, r_{+}^{1 / 2}(p ; z)\left(-V_{-}\right)(p) r_{+}^{1 / 2}(p ; z)\right)=n\left(1,\left(-V_{-}\right)^{1 / 2}(p) r_{+}(p ; z)\left(-V_{-}(p)\right)^{1 / 2}\right) .
$$

Учитывая равенства (17) и (18), получим (16). Лемма доказана.

Следующая лемма доказывается аналогично лемме 4.

Лемма 5. Для любого $z>M(p)$ оператор $V_{+}^{1 / 2}(p) r_{-}(p ; z) V_{+}^{1 / 2}(p)$ является компактным $u$

$$
N_{+}(p ; z)=n\left(-1,-V_{+}^{1 / 2}(p) r_{-}(p ; z) V_{+}^{1 / 2}(p)\right) .
$$

Теперь мы готовы к доказательству следующей леммы.

Лемма 6. Для любого $p \in \mathbb{T}^{3}$ оператор $h(p)$ имеет не более двух (не более одного) простых собственных значений, лежащих левее $m(p)($ правее $M(p))$.

ДокАЗАТЕЛьство. В силу леммы 2 имеем

$$
\begin{gathered}
n\left(1,\left(-V_{-}\right)^{1 / 2}(p) r_{+}(p ; z)\left(-V_{-}\right)^{1 / 2}(p)\right) \leqslant 2, \\
n\left(-1,-V_{+}^{1 / 2}(p) r_{-}(p ; z) V_{+}^{1 / 2}(p)\right) \leqslant 1 .
\end{gathered}
$$

Из лемм 4 и 6 , а также последних неравенств вытекает, что

$$
N_{-}(p ; z) \leqslant 2, \quad z<m(p), \quad \text { и } \quad N_{+}(p ; z) \leqslant 1, \quad z>M(p) .
$$

Лемма доказана.

Следующая лемма доказана в работах [2], [5], [14]; она описывает множество собственных значений операторов $h_{i}(p), p \in \mathbb{T}^{3}, i=1,2$.

Лемма 7. 1. Пусть $\min _{p \in \mathbb{T}^{3}} \Delta_{i}(p ; m) \geqslant 0$. Тогда для любого $p \in \mathbb{T}^{3}$ оператор $h_{i}(p), i=1,2$, не имеет собственных значений, лежащих левее точки $m$.

2. Пусть $\min _{p \in \mathbb{T}^{3}} \Delta_{i}(p ; m)<0 u \max _{p \in \mathbb{T}^{3}} \Delta_{i}(p ; m) \geqslant 0$. Тогда существует непустое открытое множество $G_{i} \varsubsetneqq \mathbb{T}^{3}$ такое, что при всех $p \in G_{i}$ оператор $h_{i}(p)$ имеет единственное собственное значение $E_{1}^{(i)}(p)$, лежащее левее $m$, а для любого $p \in \mathbb{T}^{3} \backslash G$ оператор $h_{i}(p)$ не имеет собственных значений, лежащих левее $m$.

3. Пусть $\max _{p \in \mathbb{T}^{3}} \Delta_{i}(p ; m)<0$. Тогда для любого $p \in \mathbb{T}^{3}$ оператор $h_{i}(p)$ имеет единственное собственное значение $E_{1}^{(i)}(p)$, лежащее левее $m$.

Следующая лемма доказывается аналогично лемме 7. 
Лемма 8. 1. Пусть $\max _{p \in \mathbb{T}^{3}} \Delta_{2}(p ; M) \leqslant 0$. Тогда для любого $p \in \mathbb{T}^{3}$ оператор $h_{2}(p)$ не имеет собственнъх значений, лежащих правее $M$.

2. Пусть $\max _{p \in \mathbb{T}^{3}} \Delta_{2}(p ; M)>0 u \min _{p \in \mathbb{T}^{3}} \Delta_{2}(p ; M) \leqslant 0$. Тогда существует непустое открытое множество $D \varsubsetneqq \mathbb{T}^{3}$ такое, что при всех $p \in D$ оператор $h_{2}(p)$ имеет единственное собственное значение $E_{2}(p)$, лежащее правее $M$, а для любого $p \in \mathbb{T}^{3} \backslash D$ оператор $h_{2}(p)$ не имеет собственных значений, лежащих правее $M$.

3. Пусть $\min _{p \in \mathbb{T}^{3}} \Delta_{2}(p ; M)>0$. Тогда для любого $p \in \mathbb{T}^{3}$ оператор $h_{2}(p)$ имеет единственное собственное значение $E_{2}(p)$, лежащее правее $M$.

Для доказательства теоремы 3 мы используем также следующую лемму (см. работу [14]).

Лемма 9. Для любого $p \in \mathbb{T}^{3}$ оператор $h_{1}(p)$ не имеет собственных значений, лежащих правее $M$.

\section{4. АНАЛОГ УРАВНЕНИЯ ФАДДЕЕВА ДЛЯ СОБСТВЕННЫХ ФУНКЦИЙ ОПЕРАТОРА $H$}

Введем обозначение $\overline{\mathcal{H}}=\overline{\mathcal{H}}_{0} \oplus \overline{\mathcal{H}}_{1} \oplus \overline{\mathcal{H}}_{2}$, где $\overline{\mathcal{H}}_{0}=\mathcal{H}_{0}, \overline{\mathcal{H}}_{1}=\overline{\mathcal{H}}_{2}=\mathcal{H}_{1}$. Пусть операторная матрица $T(z)$ при каждом $z \in \mathbb{C} \backslash \Sigma$ действует в пространстве $\overline{\mathcal{H}}$ по формуле

$$
T(z)=\left(\begin{array}{ccc}
T_{00}(z) & T_{01}(z) & 0 \\
T_{10}(z) & T_{11}(z) & T_{12}(z) \\
0 & T_{21}(z) & T_{22}(z)
\end{array}\right)
$$

где операторы $T_{i j}(z): \overline{\mathcal{H}}_{j} \rightarrow \overline{\mathcal{H}}_{i}, i, j=0,1,2$, задаются равенствами

$$
\begin{aligned}
\left(T_{00}(z) \psi_{0}\right)_{0} & =\left(w_{0}-z+1\right) \psi_{0}, \quad T_{01}(z) \equiv H_{01}, \\
\left(T_{10}(z) \psi_{0}\right)_{1}(p) & =-\frac{v_{0}(p) \psi_{0}}{\Delta_{2}(p ; z)}, \\
\left(T_{11}(z) \psi_{1}\right)_{1}(p) & =\frac{v_{1}(p)}{2 \Delta_{2}(p ; z)} \int \frac{v_{1}\left(q^{\prime}\right) \psi_{1}\left(q^{\prime}\right) d q^{\prime}}{w_{2}\left(p, q^{\prime}\right)-z}, \\
\left(T_{12}(z) \psi_{2}\right)_{1}(p) & =-\frac{v_{2}(p)}{\Delta_{2}(p ; z)} \int \frac{v_{1}\left(q^{\prime}\right) \psi_{2}\left(q^{\prime}\right) d q^{\prime}}{w_{2}\left(p, q^{\prime}\right)-z}, \\
\left(T_{21}(z) \psi_{1}\right)_{2}(p) & =-\frac{v_{1}(p)}{2 \Delta_{1}(p ; z)} \int \frac{v_{2}\left(q^{\prime}\right) \psi_{1}\left(q^{\prime}\right) d q^{\prime}}{w_{2}\left(p, q^{\prime}\right)-z}, \\
\left(T_{22}(z) \psi_{2}\right)_{2}(p) & =\frac{v_{2}(p)}{\Delta_{1}(p ; z)} \int \frac{v_{2}\left(q^{\prime}\right) \psi_{2}\left(q^{\prime}\right) d q^{\prime}}{w_{2}\left(p, q^{\prime}\right)-z}
\end{aligned}
$$


Отметим, что $T_{i j}(z), i, j=1,2$, при каждом $z \in \mathbb{C} \backslash \Sigma$ являются операторами Гильберта-Шмидта, а операторы $T_{00}(z), T_{01}(z)$ и $T_{10}(z)$ одномерные, следовательно, $T(z)$ - компактный оператор.

Следующая лемма устанавливает связь между собственными значениями операторов $H$ и $T(z)$.

Лемма 10. Пусть выполнено условие из $n$. 2.2. Число $z \in \mathbb{C} \backslash \Sigma$ является собственным значением оператора $H$ тогда и только тогда, когда оператор $T(z)$ имеет собственное значение, равное единище.

ДоказАтельство. Пусть $z \in \mathbb{C} \backslash \Sigma$ - собственное значение оператора $H$ и $f=$ $\left(f_{0}, f_{1}, f_{2}\right) \in \mathcal{H}$ - соответствующая собственная вектор-функция. Тогда $f_{0}, f_{1}$ и $f_{2}$ удовлетворяют системе уравнений

$$
\begin{aligned}
\left(\left(H_{00}-z I_{0}\right) f_{0}\right)_{0}+\left(H_{01} f_{1}\right)_{0} & =0 \\
\left(H_{10} f_{0}\right)_{1}(p)+\left(\left(H_{11}-z I_{1}\right) f_{1}\right)_{1}(p)+\left(H_{12} f_{2}\right)_{1}(p) & =0 \\
\left(H_{21} f_{1}\right)_{2}(p, q)+\left(\left(H_{22}-z I_{2}\right) f_{2}\right)_{2}(p, q) & =0
\end{aligned}
$$

где $I_{i}$ - единичный оператор в $\mathcal{H}_{i}, i=0,1,2$. Так как $z \notin \Sigma$, из третьего уравнения системы имеем

$$
f_{2}(p, q)=\frac{v_{2}(p) \bar{f}_{2}(q)+v_{2}(q) \bar{f}_{2}(p)}{w_{2}(p, q)-z}-\frac{1}{2} \frac{v_{1}(p) f_{1}(q)+v_{1}(q) f_{1}(p)}{w_{2}(p, q)-z},
$$

где

$$
\bar{f}_{2}(p)=\int v_{2}\left(q^{\prime}\right) f_{2}\left(p, q^{\prime}\right) d q^{\prime}
$$

Подставляя выражение для $f_{2}(p, q)$ во второе уравнение системы и равенство (21), учитывая условие из п. 2.2, получим, что система уравнений (19) имеет нетривиальные решения тогда и только тогда, когда система уравнений

$$
\begin{aligned}
f_{0} & =\left(w_{0}-z+1\right) f_{0}+\int v_{1}\left(q^{\prime}\right) f_{1}\left(q^{\prime}\right) d q^{\prime}, \\
\Delta_{2}(p ; z) f_{1}(p) & =-v_{0}(p) f_{0}+\frac{1}{2} \int \frac{v_{1}\left(q^{\prime}\right) f_{1}\left(q^{\prime}\right) d q^{\prime}}{w_{2}\left(p, q^{\prime}\right)-z}-v_{2}(p) \int \frac{v_{1}\left(q^{\prime}\right) \bar{f}_{2}\left(q^{\prime}\right) d q^{\prime}}{w_{2}\left(p, q^{\prime}\right)-z}, \\
\Delta_{1}(p ; z) \bar{f}_{2}(p) & =-\frac{v_{1}(p)}{2} \int \frac{v_{2}\left(q^{\prime}\right) f_{1}\left(q^{\prime}\right) d q^{\prime}}{w_{2}\left(p, q^{\prime}\right)-z}+v_{2}(p) \int \frac{v_{2}\left(q^{\prime}\right) \bar{f}_{2}\left(q^{\prime}\right) d q^{\prime}}{w_{2}\left(p, q^{\prime}\right)-z},
\end{aligned}
$$

эквивалентная уравнению

$$
\psi=T(z) \psi
$$

имеет нетривиальное решение (здесь мы использовали тот факт, что $\Delta_{i}(p ; z) \neq 0$ при $z \notin \Sigma, i=1,2)$. Лемма доказана.

Уравнение (22) - это аналог уравнения Фаддеева для собственных функций опеpaтора $H$. 


\section{5. ДОКАЗАТЕЛЬСТВО ОСНОВНЫХ РЕЗУЛЬТАТОВ}

5.1. Доказательство теоремы 1. Мы докажем эту теорему, считая, что выполнено условие из п. 2.2. При этом имеет место равенство

$$
\Sigma=\bigcup_{i=1}^{2} \bigcup_{p \in \mathbb{T}^{3}} \sigma_{\text {disc }}\left(h_{i}(p)\right) \cup[m ; M] .
$$

Докажем, что $\Sigma=\sigma_{\text {ess }}(H)$.

Включение $\Sigma \subset \sigma_{\text {ess }}(H)$ вытекает из критерия Вейля [11].

После того как мы получили компактное уравнение Фаддеева для собственной вектор-функции, обратное включение $\sigma_{\mathrm{ess}}(H) \subset \Sigma$ следует из общей теории Фредгольма [11]. Действительно, $T(z)$ - компактная операторнозначная аналитическая функция в $\mathbb{C} \backslash \Sigma$ и оператор $\mathcal{I}-T(z)$ обратим при больших $|z|$, где $z$ - вещественное число (здесь $\mathcal{I}$ - единичный оператор в $\overline{\mathcal{H}}$ ). Согласно аналитической теореме Фредгольма (см. книгу [11], теорема XIII.13) существует дискретное множество $S \subset \mathbb{C} \backslash \Sigma$ (возможно, пустое) такое, что операторнозначная функция $(\mathcal{I}-T(z))^{-1}$ существует и аналитична в $\mathbb{C} \backslash \Sigma$ всюду, за исключением дискретного множества $S$ (возможно, пустого), где она имеет вычеты конечного ранга. Следовательно, в силу леммы 10 у оператора $H-z \mathbf{I}$, где $\mathbf{I}$ - единичный оператор в $\mathcal{H}$, существует ограниченный обратный оператор. Если множество $\sigma(H) \backslash \Sigma$ непусто, то оно состоит из изолированных точек конечной кратности, причем это множество не имеет предельных точек, лежащих в $\mathbb{C} \backslash \Sigma$, иными словами, $(\mathbb{C} \backslash \Sigma) \cap \sigma_{\text {ess }}(H)=\varnothing$. Отсюда имеем $\sigma_{\text {ess }}(H) \subset \Sigma$. Теорема 1 доказана.

5.2. Доказательство теоремы 2. В силу леммы 6 для любого $p \in \mathbb{T}^{3}$ оператор $h(p)$ имеет не более трех простых собственных значений, лежащих вне существенного спектра этого оператора. Тогда в силу теоремы о спектре разложимых операторов из равенства (6) вытекает, что множество $\sigma_{\text {two }}(H)$ состоит из объединения не более чем трех отрезков. Использование теоремы 1 завершает доказательство теоремы 2.

5.3. Доказательство теоремы 3. Докажем часть 2 этой теоремы, части 1 и 3 доказываются аналогично.

Пусть

$$
\min _{p \in \mathbb{T}^{3}} \Delta_{1}(p ; m) \geqslant 0, \quad \min _{p \in \mathbb{T}^{3}} \Delta_{2}(p ; M) \leqslant 0, \quad \max _{p \in \mathbb{T}^{3}} \Delta_{2}(p ; M)>0 .
$$

Из первого неравенства в силу утверждения 1 леммы 7 и леммы 9 вытекает, что для любого $p \in \mathbb{T}^{3}$ оператор $h_{1}(p)$ не имеет собственных значений, лежащих левее $m$ и правее $M$. Следовательно, $\bigcup_{p \in \mathbb{T}^{3}} \sigma_{\text {disc }}\left(h_{1}(p)\right) \subset[m ; M]$.

Пусть теперь выполнены второе и третье неравенства в (23). Тогда по лемме 8 существует непустое открытое множество $D \varsubsetneqq \mathbb{T}^{3}$ такое, что для любого $p \in D$ оператор $h_{2}(p)$ имеет единственное собственное значение $E_{2}(p)$, лежащее правее $M$.

Так как функции $w_{1}(\cdot), v_{2}(\cdot)$ и $w_{2}(\cdot, \cdot)$ являются непрерывными в своих областях определения, функция $E_{2}(\cdot)$, сопоставляющая элементу $p \in D$ собственное 
значение $E_{2}(p)$, также непрерывна в $D$. Для любого $p \in \mathbb{T}^{3}$ оператор $h_{2}(p)$ ограничен и $\mathbb{T}^{3}$ - компактное множество, поэтому существует положительное число $C$ такое, что $\sup _{p \in \mathbb{T}^{3}}\left\|h_{2}(p)\right\| \leqslant C$, следовательно, для любого $p \in \mathbb{T}^{3}$

$$
\sigma\left(h_{2}(p)\right) \subset[-C ; C] .
$$

Для любого $p \in \partial D=\left\{p \in \mathbb{T}^{3}: \Delta_{2}(p ; M)=0\right\}$ существует последовательность $\left\{p_{n}\right\} \subset D$ такая, что $p_{n} \rightarrow p$ при $n \rightarrow \infty$. Положим $E_{2}^{(n)}=E_{2}\left(p_{n}\right)$. Тогда по лемме 8 для любого $\left\{p_{n}\right\} \in D$ выполняется неравенство $E_{2}^{(n)}>M$. Используя включение (24), получим, что $\left\{E_{2}^{(n)}\right\} \subset[M ; C]$. Не нарушая общности, предположим, что последовательность $\left\{E_{2}^{(n)}\right\}$ сходится при $n \rightarrow \infty$ к некоторому $E_{2}^{(0)} \in[M ; C]$ (можно взять подпоследовательность). Из непрерывности функции $\Delta_{2}(\cdot ; \cdot)$ на $\mathbb{T}^{3} \times[M ;+\infty)$ и сходимостей $p_{n} \rightarrow p, E_{2}^{(n)} \rightarrow E_{2}^{(0)}$ при $n \rightarrow \infty$ следует, что

$$
0=\lim _{n \rightarrow \infty} \Delta_{2}\left(p_{n} ; E_{2}^{(n)}\right)=\Delta_{2}\left(p ; E_{2}^{(0)}\right) .
$$

Для любого $p \in \mathbb{T}^{3}$ функция $\Delta_{2}(p ; \cdot)$ монотонно убывает на $[M ;+\infty)$ при $p \in \partial D$, отсюда мы получаем, что $\Delta_{2}\left(p ; E_{2}^{(0)}\right)=0$ тогда и только тогда, когда $E_{2}^{(0)}=M$.

Для любого $p \in \partial D$ положим $E_{2}(p)=\lim _{\substack{p^{\prime} \rightarrow p, p^{\prime} \in D}} E_{2}\left(p^{\prime}\right)=M$. Так как функция $E_{2}(\cdot)$ непрерывна на компактном множестве $D \cup \partial D$ и $E_{2}(p)=M$ при всех $p \in \partial D$, множество $\operatorname{Im} E_{2}$ значений функции $E_{2}(\cdot)$ есть отрезок $\left[M ; b_{3}\right], b_{3}>M$. Следовательно, множество $\left\{z \in \sigma_{\text {two }}(H), z \geqslant M\right\}$ совпадает с множеством $\operatorname{Im} E_{2}=$ $\left[M ; b_{3}\right]$. Таким образом, если выполнены неравенства $(23)$, то

$$
\sigma_{\mathrm{ess}}(H) \cap[M ;+\infty)=\left[M ; b_{3}\right] .
$$

Далее, доказательства утверждений 2.1-2.3 теоремы получаются из доказательства теоремы 1.4 в работе [2], если заменить $[m ; M]$ на $\left[m ; b_{3}\right]$. Теорема доказана.

Благодарности. Автор благодарит рецензента за ценные и полезные замечания. Работа частично поддержана международным Центром теоретической физики им. Абдуса Салама (Италия, Триест), и автор выражает свою признательность центру за гостеприимство и поддержку, а также благодарит Международное математическое общество за дорожный грант.

\section{Список литературы}

[1] Т. Х. Расулов, Изв. вузов. Матем., 2008, № 12, 59-69.

[2] Т. Х. Расулов, Матем. заметки, 83:1 (2008), 86-94.

[3] С. Н. Лакаев, Т. Х. Расулов, Матем. заметки, 73:4 (2003), 556-564.

[4] Т. Х. Расулов, ТМФ, 152:3 (2007), 518-527.

[5] S. Albeverio, S. N. Lakaev, T. H. Rasulov, J. Stat. Phys., 127:2 (2007), 191-220.

[6] Г. Р. Ёдгоров, М. Э. Муминов, ТМФ, 144:3 (2005), 544-554.

[7] Т. Х. Расулов, ТМФ, 161:2 (2009), 164-175.

[8] R. A. Minlos, H. Spohn, "The three-body problem in radioactive decay: the case of one atom and at most two photons", Topics in Statistical and Theoretical Physics, Amer. Math. Soc. Transl. Ser. 2, 177, ред. R. L. Dobrushin, R. A. Minlos, M. A. Shubin, A. M. Vershik, AMS, Providence, RI, 1996, 159-193. 
[9] Ю. В. Жуков, Р. А. Минлос, ТМФ, 103:1 (1995), 63-81.

[10] Г. М. Жислин, Труды ММО, 9, 1960, 81-120.

[11] М. Рид, Б. Саймон, Методъ современной математической физики, т. 4, Анализ операторов, Мир, М., 1982.

[12] С. Албеверио, С. Н. Лакаев, Ж. И. Абдуллаев, Функи. анализ и его прил., 36:3 (2002), $56-60$.

[13] S. Albeverio, S. N. Lakaev, Z. I. Muminov, Math. Nachr., 280:7 (2007), 699-716; arXiv: math-ph/0312050.

[14] S. Albeverio, S. N. Lakaev, R. Kh. Djumanova, Rep. Math. Phys., 63:3 (2009), 359-380; arXiv: math-ph/0501024.

[15] П. Халмош, Гильбертово пространство в задачах, Мир, М., 1970.

Поступила в редакцию 1.12.2009, после доработки 31.01.2010 